\title{
WFPC2 Studies of the Disk and Jet of HH 30
}

\author{
Karl Stapelfeldt \\ Jet Propulsion Laboratory, Calif. Institute of Technology
}

Chris Burrows, John Krist

Space Telescope Science Institute

\author{
Alan Watson \\ New Mexico State University \\ and the WFPC2 Science Team
}

\begin{abstract}
Hubble Space Telescope imaging of $\mathrm{HH} 30$ has revealed this object to be a prototype young stellar object (YSO) accretion disk system. An optically thick circumstellar absorption disk, $450 \mathrm{AU}$ in diameter, is seen extending perpendicular to highly collimated bipolar jets. The jet width is resolved in the transverse direction and increases with distance from the central star. The size and age of the disk compare well to the early solar nebula during planet formation. HH 30 is the first YSO disk ever observed at $14 \mathrm{AU}$ resolution, and the first viewed close enough to edge-on that the vertical structure of the disk is clearly visible. These HST images of HH 30 offer the first-ever opportunity to directly measure the flaring profile of an astrophysical accretion disk. We have compared the images to model nebulosities calculated in both multiple and single scattering regimes, in the latter case via chi-squared minimization. This analysis constrains the circumstellar density distribution and grain scattering properties. A brief summary of the results is presented here; full details and references appear in Burrows et al., Ap.J. (1996).
\end{abstract}

\section{Introduction}

HH 30 is a nebulous low-mass star in the Taurus star forming cloud, located near HL Tauri. The system is composed of a young stellar object and its associated emission and reflection nebulae. The emission nebulae have the form of two collimated jets located on opposite sides of the star and flowing nearly in the plane of the sky. The stellar component is quite faint. No reliable extinction estimate exists, and thus the stellar luminosity is not well constrained. Far infrared and millimeter wavelength excess emission have been measured for $\mathrm{HH}$ 30 , but the inferred circumstellar mass is small $\left(\approx 10^{-3} \mathrm{M}_{\odot}\right)$. Existing data only poorly constrain the infrared spectral energy distribution. 


\section{Observations \& Results:}

Observations in V, R, and I band filters were made 10 months apart in 1994 and 1995. At the distance of $\mathrm{HH} 30$, the WFPC2 cameras in wide-field mode provide a spatial resolution of $14 \mathrm{AU}$ per pixel. These images (see Fig. 1) reveal the following facts about $\mathrm{HH} 30$ :

- The central star is not directly seen - HH 30 is entirely nebulosity at optical wavelengths

- The HH 30 nebulosities are clearly resolved into 5 components:

1. A bright, highly collimated emission line jet extending NNE

2. A faint counter-jet extending SSW

3. A bright reflection nebula at the base of the bright jet which extends symmetrically $450 \mathrm{AU}$ across the jet axis

4. A faint reflection nebula at the base of the counter-jet

5. A dark absorption lane at the center of the system, separating the two jets and reflection nebulae from each other

- The central illuminating source is variable: at the second epoch, the reflection nebulae had faded in brightness by $40 \%$.

- The bright blueshifted jet has a FWHM less than $25 \mathrm{AU}$ at the base of the reflection nebula and an opening angle of 3 degrees within $700 \mathrm{AU}$ of the star. The ionized jet is much narrower than the cleared region revealed by the reflection nebula, indicating that the collimation must occur very close to the star.

- Knots in the jet and counter-jet have oppositely directed proper motions corresponding to pattern speeds ranging from $100-300 \mathrm{~km} \mathrm{~s}^{-1}$

- The typical jet knot spacing is $100 \mathrm{AU}$, which combined with the proper motions suggests that new knots emerge from the source every few years.

\section{Modeling the Reflection Nebulosity}

Dust in the circumstellar disk reflects light from the central star and produces the observed reflection nebulosities at the disk's upper and lower surfaces. Model nebulae can be calculated for a specified density distribution. $\lambda=0.8 \mu \mathrm{m}$ is the preferred wavelength to calculate nebula models, as the HST I band image is predominantly reflection nebulosity with little contribution from jet emission. We make the following assumptions:

Axisymmetric density distribution:

A parameterized surface density $\Sigma(\mathrm{r})=\Sigma_{0}\left(\mathrm{r} / \mathrm{r}_{0}\right)^{-p}$; a gaussian vertical profile $\rho(\mathrm{z})=\rho_{0} \exp \left(-\mathrm{z}^{2} / \mathrm{H}(\mathrm{r})^{2}\right)$ valid for a vertically isothermal, hydrostatic, non-self-gravitating disk; a parameterized scale height $\mathrm{H}(\mathrm{r})=\mathrm{H}_{0}\left(\mathrm{r} / \mathrm{r}_{0}\right)^{\beta}$; and 


\section{HST Image of HH 30}

(Burrows, Stapelfeldt, Watson, Krist, et al. 1996)
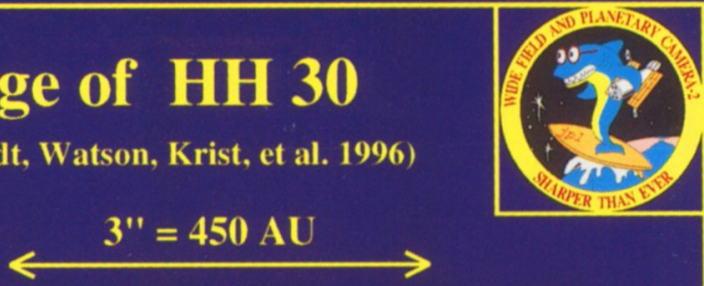
disk mass $\mathrm{M}_{d}$, inner and outer radii $\mathrm{R}_{i} \approx 0.5 \mathrm{AU}$ and $\mathrm{R}_{o}=250 \mathrm{AU}$. For the reference radius $r_{0}$ we choose $100 \mathrm{AU}$. These lead to the density law

$$
\rho(r, z)=\frac{(2-p)}{2 \pi^{3 / 2}} \frac{M_{d} R_{o}^{-2} H^{-1}(r)}{\left(1-\left(\frac{R_{i}}{R_{o}}\right)^{(2-p)}\right)}\left(\frac{r_{0}}{r}\right)^{p} \exp \left(-z^{2} / H(r)^{2}\right)
$$

Adopted optical properties for the dust grains:

Invariant grain opacity $\kappa=120 \mathrm{~cm}^{2} \mathrm{gm}^{-1}$, appropriate to interstellar grains at $\lambda=0.8 \mu \mathrm{m}$; invariant grain albedo $a=0.5$; and a Henyey-Greenstein scattering phase function, characterized by the asymmetry parameter $g=0.29$ for interstellar grains at $\lambda=0.8 \mu \mathrm{m}$.

Two computational strategies were taken to simulate the nebulosity produced when the above density distribution is illuminated by the central star:

1. Monte Carlo simulations of multiple scattering: In this approach, individual photons are launched and propagated through the density distribution. Scattering angles and optical depths are selected randomly over the appropriate distribution functions. Useful runs take a few hours on a single-processor DEC Alpha workstation.

2. Single scattering model: In this approach, the nebula brightness contribution is calculated for each volume element in the density distribution and then projected onto the sky. The local brightness contribution is weighted according its opacity, the inverse square law, the grain phase function, and the extinction toward the star and toward the observer. Computationally this is much faster; about 2 minutes are required per model run, allowing $\chi^{2}$ minimization to be implemented to determine the best-fit model.

Comparison of the multiple scattering and single scattering results shows that the latter is an adequate description of the problem. Model parameters to determine via comparison to the WFPC2 images are the disk inclination $i$; the disk mass $\mathrm{M}_{d}$; the scale height radial power law index $\beta ; \mathrm{H}_{0}$, the scale height normalization at reference radius $\mathrm{r}_{0}=100 \mathrm{AU}$; and $p$, the surface density radial power law index.

\section{Conclusions from the modeling work:}

- An observing latitude $i=6-8$ degrees is needed to match the brightness ratio of the reflection nebulosities. This is somewhat larger than the inclination inferred from the jet radial velocities and proper motions.

- Forward scattering dust grains are needed to reproduce the central brightening of the reflection nebulosities. For a H-G phase function the best-fit asymmetry parameter $g=0.64$, which is consistent with more recent determinations of the ISM value at $\lambda=0.8 \mu \mathrm{m}$.

- The disk mass is small. Models with masses greater than $>10^{-3} \mathrm{M}_{\odot}$ produce an absorption lane that is too wide for nearly all choices of the other model parameters. The best fit mass is $4 \times 10^{-4} \mathrm{M}_{\odot}$ of dust+gas. This is a lower limit, but it is consistent with the disk mass inferred from the millimeter continuum emission. 
- The disk surface density profile is constrained by the extinction we adopt toward the central star; $A_{\lambda=0.8 \mu m}$ must exceed 24 mag in order to account for the faint $\mathrm{K}$ magnitude of the star. Flat surface density profiles do not produce enough extinction, and the star becomes directly visible in such models. Steeper surface density profiles place more material closer to the star, providing more extinction along the line of sight through the disk for a fixed disk mass. Our best-fit solution has $\mathrm{p}=0.8$

- The scale height radial power law index is not uniquely determined. There is a geometrical degeneracy between the two parameters in the scale height law $\mathrm{H}(\mathrm{r})=\mathrm{H}_{0}\left(\mathrm{r} / \mathrm{r}_{0}\right)^{\beta}$ : larger $\beta$ can be counteracted by a smaller $\mathrm{H}_{0}$ over the observed range of radii. There is simultaneously an "extinction" degeneracy between $\beta$ and $p$ : to maintain the same column density (extinction) toward the star, $p$ must increase as $\beta$ increases and vice-versa. These interacting parameters produce a family of solutions with comparable $\chi^{2}$ values. Acceptable fits give $1.2 \leq \beta \leq 1.5$, while $16.5 \mathrm{AU} \geq \mathrm{H}_{0} \geq 15 \mathrm{AU}$.

\section{Relating the $\mathrm{HH} 30$ image fitting results to accretion disk theory}

- The fitted scale height determines the local dynamical temperture in the case of hydrostatic equilibrium. The fitting results for $\mathrm{H}_{0}$ imply

$$
\begin{aligned}
& H(r)=H_{0}\left(r / r_{0}\right)^{\beta}=\sqrt{\frac{k T(r) r^{3}}{G M m}} \quad T=T_{0}\left(r / r_{0}\right)^{-q} \quad q=3-2 \beta \\
& \left.\mathrm{T}_{0}=50 \mathrm{M}\left(\mathrm{M}_{\odot}\right)\left(\mathrm{H}_{0} \mathrm{AU}\right) / 15.5\right)^{2} \\
& \text { for } \mathrm{M}=0.67 \mathrm{M}_{\odot} \text { and } \mathrm{H}_{0}=15.5, \mathrm{~T}_{0}=34 \mathrm{~K} \text { at } \mathrm{r}_{0}=100 \mathrm{AU}
\end{aligned}
$$

- The fitting results for the flaring exponent $\beta$ constrain the temperature power law index $q$ to lie within the range $0.6>q>0.0$. Theory predicts $q=0.75(\beta=1.125)$ in the case of a steady-state accretion disk or for a geometrically thin passive reprocessing disk. Such models produce a nebula that is too flat, and are not consistent with the images of $\mathrm{HH} 30$.

- Additional theoretical insights lead to the choice of a preferred value for the scale height power law index $\beta$. The low disk mass determines that the disk is optically thin to its own thermal emission. Such a disk, if hydrostatically supported and in radiative thermal equilibrium with the central star, is consistent with the image fitting results only when $\beta=1.45$. In this case, the disk temperature declines slowly with radius because the system absorbs visible starlight much more efficiently than it can re-radiate the energy away in the infrared.

- Accretion should consume the HH 30 disk within $10^{4}-10^{5}$ years. This estimate is simply the small disk mass $\left(<10^{-3} \mathrm{M}_{\odot}\right)$ divided by the expected mass accretion rate $\left(>10^{-8} \mathrm{M}_{\odot} \mathrm{yr}^{-1}\right)$. Thus we view this system near the end of its evolution. The remaining disk mass is perhaps a factor of 10 smaller than the minimum-mass solar nebula.

Acknowledgments. This work was performed under contract to NASA as part of the WFPC2 GTO science program. 